\title{
Evaluación de la Actividad Anti-inflamatoria de Propóleos Chileno sobre Cortes Histológicos de Orejas de Ratón
}

\author{
Evaluation of the Anti-inflammatory Activity of Chilean \\ Propolis on Histological Sections of Mouse Ears
}

Daniela Núñez R. ${ }^{1}$; Natalia Balboa P. ${ }^{2}$; Marysol Alvear Z. ${ }^{2}$; Anabelle Ceron N. ${ }^{3}$; Karen Abarzua S. ${ }^{1}$ \& Adriana Vasconcellos C. ${ }^{1}$

NÚÑEZ, R. D.; BALBOA, P. N.; ALVEAR, Z. M.; CERON, A. N.; ABARZUA, S. K. \& VASCONCELLOS, C. A. Evaluación de la actividad anti-inflamatoria de propóleos chileno sobre cortes histológicos de orejas de ratón. Int. J. Morphol., 36(1):189-193, 2018.

RESUMEN: El propóleos es un producto resinoso complejo producido por las abejas Apis mellifera, el cual posee diversas actividades biológicas como inmunomodulador, antiinflamatorio, anticancerígeno, antiviral, antibacteriano, antioxidante, entre otros. El propósito del siguiente estudio fue realizar una evaluación in vivo de las propiedades antiinflamatorias de un extracto de propóleos chileno, sobre el modelo de edema auricular inducido por 13-acetato-12-O-tetradecanoilforbol (TPA) en pabellón auricular de ratón, para posterior evaluación y análisis histológico. El extracto de propóleos chileno (EEP) utilizado se obtuvo a partir de un macerado etanólico, rotaevaporado y liofilizado. Se observó que el EEP disminuyó el edema y el infiltrado inflamatorio de forma significativa. Estos resultados sugieren que el extracto etanólico de propóleos chileno posee potenciales efectos antiinflamatorios o moduladores del sistema inmunológico en edema auricular.

PALABRAS CLAVE: Inflamación; Propóleos chileno; Productos naturales; Leucocitos.

\section{INTRODUCCIÓN}

El propóleos es una sustancia de aspecto resinoso fabricado por la abeja (Apis mellifera) a partir de las yemas de plantas adyacentes a la colmena (Ngatu et al., 2011). Las abejas mezclan estas sustancias obtenidas de los árboles con su saliva y forman el propóleos, el cual es utilizado para sellar los orificios presentes en la colmena y evitar de esta forma el ingreso de microorganismos, además de ser principalmente un antimicrobiano de origen natural (Abu-Mellal et al., 2012). Dentro de los componentes del propóleos se encuentran flavonoides, ácidos orgánicos, fenoles, diversas enzimas, vitaminas y minerales (Benkovic et al., 2007). Siendo conformado por un $50 \%$ de resina, $30 \%$ de saliva, $10 \%$ de aceites aromáticos y esenciales, $5 \%$ de polen y $5 \%$ de otras sustancias que incluyen vitaminas como A, B (1, 2 y 6), C, D y E, y otros como trazas de minerales. Dentro de estos componentes, debemos mencionar los polifenoles, estos se pueden agrupar dependiendo del número de anillos fenólicos, los cuáles pueden interactuar con anillos fenólicos adyacentes. Estos compuestos comprenden: flavonoides, ácidos fenólicos, taninos, estilbenos y lignanos (Ignat et al., 2011). Dentro de los polifenoles podemos subdividirlos en dos grandes grupos: flavonoides y no flavonoides. En el grupo de los flavonoides se encuentran las antocianinas, flavonoles, flavanonas, flavonas, isoflavonas, proantocianidinas (taninos condensados), entre otras (Chang et al., 2002; Takos et al., 2006). En los no flavonoides se encuentran los alcoholes monofenólicos, ácidos fenólicos simples y estilbenos. Debido a esta variedad de compuestos, el propóleos ha sido utilizado por la medicina tradicional, debido a la presencia de diversidad de propiedades como antibacterial, antifúngico, antiviral, anti-inflamatorio, inmuno-estimulante y en el tratamiento de patologías gastrointestinales, como úlceras (Erejuwa et al., 2012; Mihai et al., 2012; Valenzuela et al., 2015). El propósito del siguiente trabajo, fue evaluar in vivo la actividad anti-inflamatoria de un propóleos chileno recolectado en la localidad de Mulchén, región del Bio-Bio, en el modelo de edema auricular de ratón inducido por TPA y el análisis histológico de estas muestras.

\footnotetext{
${ }^{1}$ Depto. de Ciencias Básicas. Facultad de Medicina, Universidad de La Frontera, Chile.

${ }^{2}$ Depto. de Ciencias Químicas y Recursos Naturales. Facultad de Ingeniería y Ciencias, Universidad de La Frontera, Chile.

${ }^{3}$ Facultad de Ciencias Químicas, Universidad La Salle, México.
} 


\section{MATERIAL Y MÉTODO}

Recolección de la muestra de propóleos. La recolección de la muestra de propóleos chileno fue obtenida desde los campos alrededor (100 kilómetros a la redonda) de la ciudad de Mulchén (Región Bio-Bío, Chile). La muestra fue obtenida con el método tradicional de raspado con espátula de los panales de abeja. Posteriormente la muestra fue triturada y tamizada para eliminar la madera y residuos de insectos.

Preparación de extracto de polifenoles de propóleos. La muestra de propóleos fue almacenada a $4{ }^{\circ} \mathrm{C}$ protegida de la luz, previo al análisis. $15 \mathrm{~g}$ de propóleos fueron mezclados con $50 \mathrm{~mL}$ de etanol absoluto, grado analítico (Merck). La muestra fue macerada durante 7 días, con dos periodos de agitación durante dos horas al día. Terminado los 7 días de maceración, la muestra fue filtrada con papel filtro Whatman $\mathrm{N}^{\circ}$ 1, método modificado de Barrientos et al. (2013).

Cuantificación de polifenoles y flavonoides. La determinación de polifenoles y flavonoides totales presentes en el extracto etanólico de propóleos se realizó de acuerdo al método descrito por Lillo et al. (2016).

Animales de experimentación. Se utilizaron ratones machos adultos CD-I (25-30 g de peso corporal), obtenidos desde el bioterio de la Facultad de Ciencias Químicas de la Universidad de La Salle, México. Estos se mantuvieron en un ambiente controlado, con ciclos diarios de 12 horas de luz/oscuridad, temperatura ambiental de $22^{\circ} \mathrm{C}$ y comida y agua ad libitum.

Edema auricular en ratón inducido con TPA. El edema auricular se indujo de acuerdo al método descrito por De Young (1989) y modificado por Payá et al. (1992). Los animales fueron pesados y sedados mediante una dosis de pentobarbital sódico IP (35-70 mg/kg PV). Comprobada su sedación, se procedió a la aplicación de una solución de TPA (2,5 $\mu \mathrm{g} / \mathrm{oreja})$ disuelto en etanol $(20 \mu \mathrm{L})$, siendo aplicado tópicamente en la superficie interna y externa del pabellón auricular de la oreja derecha, utilizando como control la oreja izquierda. A un grupo de ratones tratados con TPA, se les aplicó un tratamiento con propóleos chileno (100 $\mu \mathrm{g} /$ oreja) disuelto en etanol $(20 \mu \mathrm{L})$.Transcurridas 4 horas de aplicados los tratamientos, se sacrificaron los ratones con una sobredosis de $\mathrm{CO}_{2}$ sobre el $70 \%$, realizando el reconocimiento de los signos de muerte (cese del latido cardíaco y respiración, ausencia de reflejos ocular y descenso de la temperatura corporal). Comprobada la muerte de los animales, se procedió a la obtención de las muestras de cada pabellón auricular por sacabocado ( $6 \mathrm{~mm}$ de diámetro), siendo depositadas en formol al 10\% a pH 7,4 por 48 horas.
Histopatología. Una vez fijadas las muestras en formol, fueron lavadas en agua para eliminar el exceso de fijador. A continuación, se realizaron los cortes en un micrótomo de congelación marca Leitz, con un grosor de $40 \mu \mathrm{m}$. las muestras fueron montadas en portaobjetos para el consiguiente estudio histológico. A continuación, se realizó la fijación de las muestras durante 1 minuto en metanol, y finalmente teñidas con Hematoxilina-Eosina (15 y 5 min respectivamente). Luego, los cortes fueron deshidratados con alcohol etílico en concentración ascendente $\left(50^{\circ}, 70^{\circ}, 90^{\circ}\right.$, etanol absoluto), finalizando con xilol-etanol ( $3 \mathrm{~min}$ ) y xilol (5 $\mathrm{min})$. Las muestras fueron montadas en un medio resinoso y evaluadas con un microscopio óptico marca Leica.

Se realizaron diversas fotografías de las muestras histológicas, las que fueron digitalizadas mediante el uso de una cámara Canon (PowerShot A810) y un microscopio marca Leica a un aumento final de 100X. Luego de la captura de las imágenes, estas fueron transferidas al programa Image-pro Plus (demo), para realizar la medición del diámetro de las muestras. Con la ayuda de un portaobjetos graduado, se tomaron 5 mediciones al azar de cada una de las muestras desde el extremo de epidermis a epidermis de forma transversal.

Análisis estadístico. Para determinar si existen diferencias significativas entre las muestras en cada uno de los análisis, se realizó en ANOVA de un factor y posteriormente un test de Tukey con un nivel de significancia del 95\%. Para el análisis estadístico se utilizó el programa Prisma 5.0 demo.

\section{RESULTADOS}

Análisis de compuestos químicos del propóleos. La cuantificación espectrofotométrica del extracto etanólico del propóleos chileno evidenció una alta concentración de polifenoles (método de Folin Ciocalteu) y flavonoides totales (métodos colorimétricos de cuantificación, $\mathrm{AlCl}_{3}$ y 2,4 Dinitrofenilhidrazina) (Tabla I). Ambas técnicas colorimétricas permiten cuantificar diferentes grupos de flavonoides: flavonas y flavonoles; flavanonas, flavanonoles e isoflavonas respectivamente, presentes en la muestra de propóleos.

Análisis histológico. El análisis histológico presentó marcadas diferencias en la evaluación de los distintos tratamientos. La muestra de pabellón auricular normal (Figs. 1 A, B y C), corresponde a una muestra control sin tratamiento. Mientras que el control de inflamación corresponde al tratamiento con TPA (Figs. 1 D, E y F), donde se observa bajo el epitelio una vasodilatación acentuada, marcado edema y 
congestión e infiltración leucocitaria inicial. Mientras que en el tratamiento de propóleos más TPA (Figs. 1 G, H y I), se observa edema y congestión moderada, con menor infiltración inflamatoria.

Tabla I. Cuantificación espectrofotométrica de polifenoles totales de propóleos chileno.

\begin{tabular}{ccc}
\hline Polifenoles Totales* $(\mathrm{mg} / \mathrm{g})$ & $\mathrm{AlCl}_{31}(\mathrm{mg})$ & $2,4-\mathrm{DNFH} 2(\mathrm{mg} / \mathrm{g})$ \\
\hline $247 \pm 9$ & $75,2 \pm 4.1$ & $118 \pm 11$ \\
\hline
\end{tabular}

Los resultados fueron expresados como el promedio \pm la desviación estándar. *Contenido de polifenoles totales $=$ equivalentes de ácido gálico $\left(\mu \mathrm{g} \mathrm{mL}^{-1}\right) \times$ volumen total del extracto etanólico $(\mathrm{L}) \div$ masa de la muestra $(\mathrm{g}) \mathrm{x}$ factor de dilución. 1Contenido de flavonas y flavonoles $=$ equivalentes de quercetina u pinocembrina $\left(\mu \mathrm{g} \mathrm{mL} \mathrm{m}^{-1}\right)$. 2Contenido de flavanonas $\mathrm{y}$ flavanonoles $=$ equivalentes de pinocembrina $\left(\mu \mathrm{g} \mathrm{mL}^{-1}\right)$.
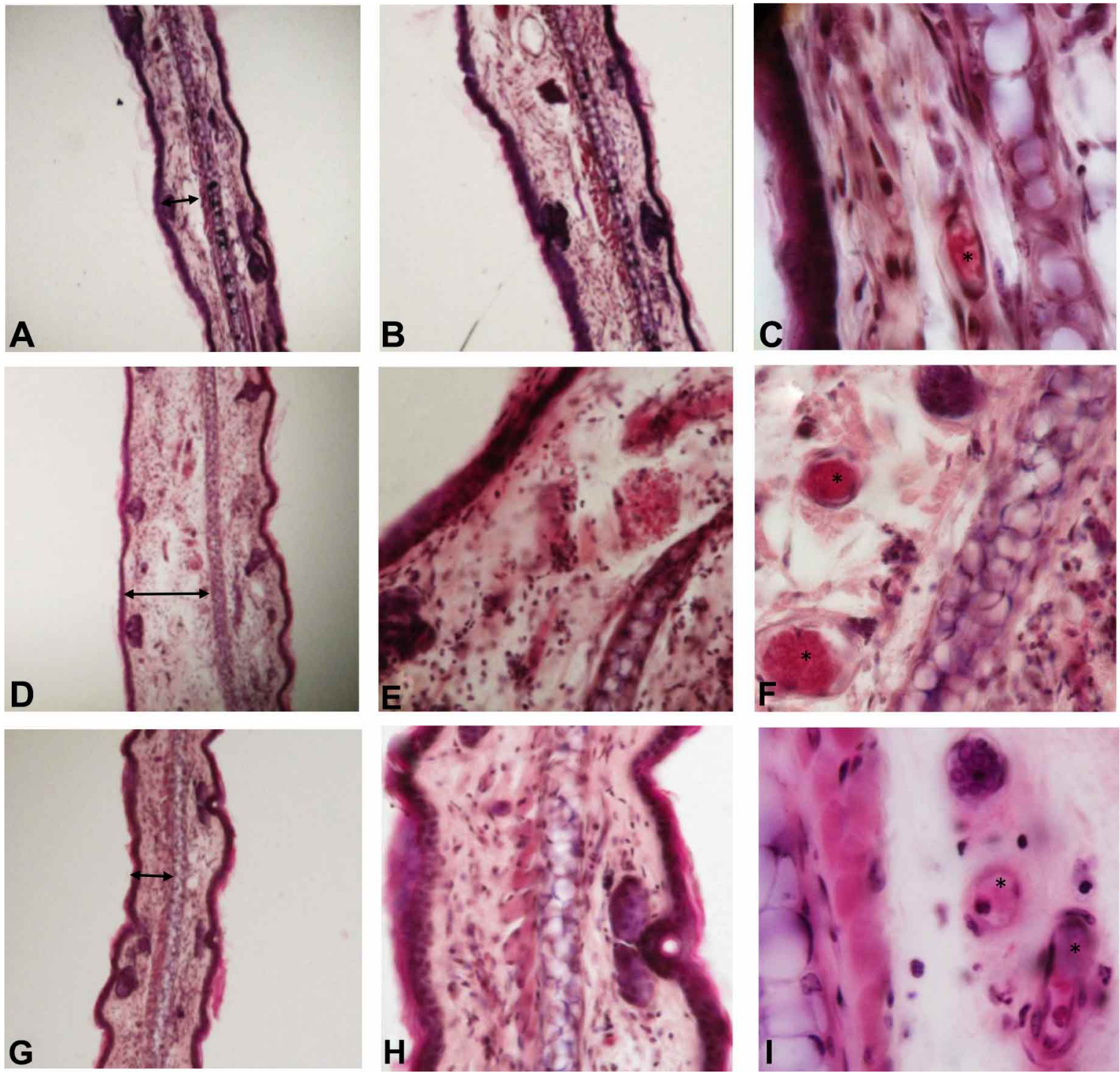

Fig. 1. Evaluación de la actividad anti-inflamatoria de propóleos chileno en cortes transversales de pabellón auricular tratados con TPA. (A, B y C) Pabellón auricular normal, control sin tratamiento. (D, E y F) pabellón tratado con inducción de inflama-

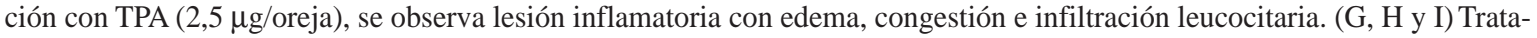
miento con propóleos chilenos (100 $\mu \mathrm{g} /$ oreja) y TPA $(2,5 \mu \mathrm{g} /$ oreja), se observa edema y congestión menos marcada, con disminución del proceso inflamatorio. Las muestras fueron teñidas con hematoxilina-eosina, el $*$ indica capilares y las flechas indican el espacio entre la epidermis y el cartílago elástico (A, D y G 100X; B, E y H 400X; C, F y I 1000X). 
Medición diámetro de muestras histológicas. Los resultados muestran diferencias significativas en la medición del diámetro de los cortes histológicos entre los tratamientos evaluados (Fig. 2). Se observó un aumento considerable del diámetro en la muestra tratada solo con TPA $(0.48 \mathrm{~mm})$, seguido por la muestra tratada con TPA más propóleos $(0.26$ $\mathrm{mm}$ ) donde se observó una reducción considerable del diámetro de la muestra, y por último la muestra control $(0.15$ $\mathrm{mm}$ ) es la que presentó el menor diámetro.

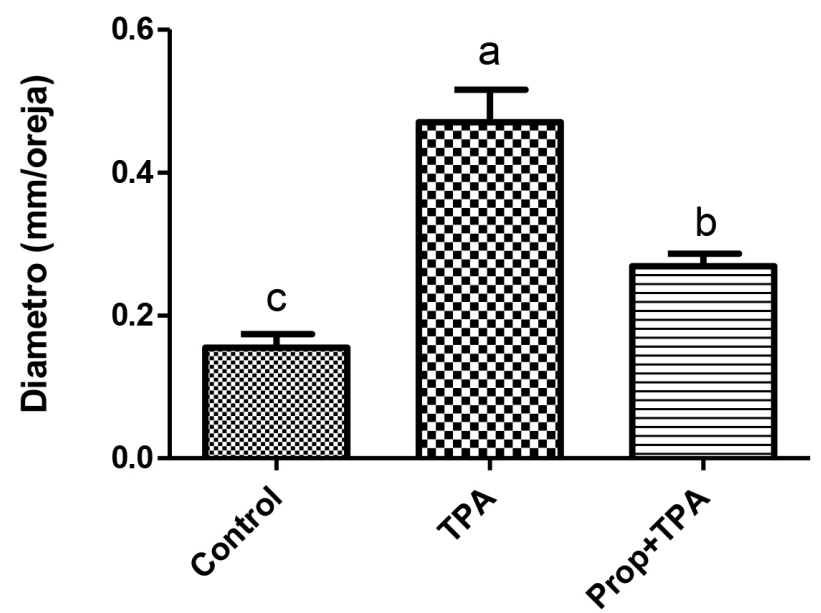

Fig. 2. Evaluación del diámetro del edema auricular de ratón inducido con TPA y tratado con propóleos chileno. Se puede observar un aumento considerable del diámetro en la muestra tratada con TPA, mientras que la muestra tratada con propóleos presenta una disminución del diámetro. Las medias con distintas letras indican diferencias significativas en la prueba de Tukey $(\mathrm{P} \leq 0,05)$. Control: Muestra sin tratamiento; TPA: Muestra tratada con TPA; Prop+TPA: Muestra tratada con extracto etanólico de propóleos y TPA.

\section{DISCUSIÓN}

La respuesta inflamatoria es una medida de protección del cuerpo ante una injuria, que permite proteger al huésped del agente agresor, con la finalidad de reestablecer la homeostasis (Moreno, 2016). Sin embargo, una respuesta descontrolada del organismo, puede generar diversos síntomas dolorosos en los pacientes (Gautam and Jachak, 2009; Zhang et al., 2017). En la constante búsqueda de nuevos fármacos con actividad antiinflamatoria, los productos naturales nos brindan una gran diversidad de fitoquímicos, con una incomparable complejidad estructural y un gran potencial biológico (Gómez et al., 2011). A nivel mundial, el propóleos ha sido utilizado por la medicina tradicional desde la antigüedad, esto gracias a sus diversas propiedades farmacológicas entre las que se destaca su potencial antiinflamatorio, sin embargo, su composición y propiedades varían ampliamente de un lugar a otro. Aunque se ha descrito la actividad antiinflamatoria de propóleos recogidos de diversas regiones del mundo, el potencial del propóleos chileno no se ha explorado como antiinflamatorio (Moreno et al., 2000; Naik et al., 2015). Siendo esta la primera evaluación in vivo con evaluación histológica que se realiza con un propóleos chileno.

Nuestros resultados obtenidos en los ensayos in vivo indicarían que el extracto de propóleos chileno, disminuiría el proceso inflamatorio agudo de forma significativa en una lesión tópica con TPA gracias a la alta concentración de polifenoles presentes en la muestra, disminuyendo el diámetro de la oreja inflamada de forma significativa. Estos resultados son similares a los presentados por Naik et al. (2015), en propóleos Hindúes, donde observaron que el extracto etanólico, sus fracciones y compuestos volátiles del propóleos presentaban actividad antiinflamatoria tópica significativa disminuyendo el diámetro de inflamación. Diversos propóleos chilenos se caracterizan por presentar una alta concentración de pinocembrina, sin embargo se presume que la sinergia de los compuestos polifenólicos debidos a la diversidad de flora adyacente a la colmena estarían involucrados en las diferentes actividades biológicas aquí reportadas (Herrera et al., 2010).

Además, nuestros resultados de la evaluación histológica nos permitieron observar los cambios en el tejido, pudiendo determinar que el tratamiento con propóleos permite el mantenimiento de la integridad del epitelio, disminuyendo la congestión y vasodilatación en pequeños vasos y el edema en el tejido conectivo, además de reducir la respuesta inflamatoria del tejido.

CONCLUSIÓN. Los resultados obtenidos en este trabajo nos permiten concluir que el extracto etanólico de propóleos chileno presenta actividad antiinflamatoria sobre lesiones tópicas con inflamación aguda generadas con TPA en pabellón auricular de ratón, disminuyendo de forma significativa el diámetro y los hallazgos histológicos de la lesión generada por el proceso inflamatorio. Por lo tanto, estos datos podrían ser utilizados para profundizar sobre el potencial del propóleos chileno en patologías asociadas a procesos inflamatorios.

AGRADECIMIENTOS. Se agradece al Departamento de Ciencias Básicas, al Departamento de Ciencias Químicas y Recursos Naturales de la Universidad de La Frontera y a la Facultad de Ciencias Químicas de la Universidad La Salle (México) por facilitar sus dependencias para este trabajo. Además, por la sustentación económica de la Beca Doctoral Nacional y Beca de Apoyo de tesis CONICYT n²1120557 y 21140816. 
NÚÑEZ, R. D.; BALBOA, P. N.; ALVEAR, Z. M.; CERON, A.; ABARZUA, S. K. \& VASCONCELLOS, C. A. Evaluation of the anti-inflammatory activity of Chilean propolis on histological sections of mouse ears. Int. J. Morphol., 36(1):189-193, 2018.

SUMMARY: Propolis is a complex resinous product produced by bees Apis mellifera, which has a number of biological activities such as an immunomodulator, anti-inflammatory, anticarcinogenic, antiviral, antibacterial, antioxidant, among others. The purpose of the following study was to perform an in vivo evaluation of the anti-inflammatory properties of a Chilean propolis extract, on the model of atrial edema induced 12-O-tetradecanoyl phorbol-13- acetate (TPA) in the mouse auricular pavilion, for later evaluation and histological analysis. The Chilean propolis extract (EPP) used was obtained from an ethanolic, rotaevaporated and lyophilized macerate. It was observed that the EPP significantly decreased edema and inflammatory infiltrate. These results suggest that the ethanolic extract of Chilean propolis possesses potential anti-inflammatory or modulatory effects of the immune system in atrial edema.

KEY WORDS: Inflammation; Chilean propolis; Natural products; Leukocytes.

\section{REFERENCIAS BIBLIOGRÁFICAS}

Abu-Mellal, A.; Koolaji, N.; Duke, R.; Tran, V. \& Duke, C. Prenylated cinnamate and stilbenes from Kangaroo Island propolis and their antioxidant activity. Phytochemistry, 77: 251-9, 2012.

Banskota, A.; Tezuka, Y.; Midorikawa, K.; Matsushige, K. \& Kadota, S. Two Novel Cytotoxic Benzofuran Derivatives from Brazilian Propolis. J. Nat. Prod., 63: 1277-9, 2000.

Barrientos, L.; Herrera, C.; Montenegro, G.; Ortega, X.; Veloz, J.; Alvear, M. \& Salazar, L. Chemical and botanical characterization of Chilean propolis and biological activity on cariogenic bacteria Streptococcus mutans and Streptococcus sobrinus. Braz. J. Microbiol., 44(2):577-85, 2013.

Benkovic, V.; Knezevic, A.; Brozovic, G.; Knezevic, F.; Dikic, D.; Bevanda, M. \& Orsolic, N. Enhanced antitumor activity of irinotecan combined with propolis and its polyphenolic compounds on Ehrlich ascites tumor in mice. Biomed. Pharmacother, 61(5):292-7, 2007.

Chang, C.; Yang, M.; Wen, H. \& Chern, J. Estimation of total flavonoid content in propolis by two complementary colorimetric methods. $J$. food and drug analysis, 10(3):178-182, 2002.

De Young, L.; Kheifets, J.; Ballaron, S. \& Young, M. Edema and cell infiltration in the phorbol ester-treated mouse ear edema are temporally separate and can be differentially modulated by pharmacological agents. Agents Actions 26:335-41, 1989.

Dulcetti, O.; Andreucci, C.; Barbosa, I.; Pulz, C.; De Oliveira, F. \& Marcucci, M. Investigation of the Anti-inflammatory and Analgesic Activities of a Sample of Brazilian Propolis. Acta Farm. Bonaerense, 23(3):285-91, 2004.

Erejuwa, O.; Sulaiman, S. \& Wahab, M. Honey-a novel antidiabetic agent. Int. J. Biol. Sci., 8(6):913-34, 2012.

Gautam, R. \& Jachak, S. Recent developments in anti-inflammatory natural products. Med. Res. Rev., 29:767-820, 2009.

Gómez, Y.; Vilvey, L.; Sánchez, L. \& Díaz L. El uso del propóleo el 5\% en el tratamiento de la alveolitis. Gaceta médica espirituana 10(1):2008.
Herrera, C.; Alvear, M.; Barrientos, L.; Montenegro, G. \& Salazar, L. The antifungal effect of six commercial extracts of Chilean propolis on Candida spp. Cien. Inv. Agr., 37(1):75-84, 2010.

Ignat, I.; Volf, I. \& Popa, V. A critical review of methods for characterisation of polyphenolic compounds in fruits and vegetables. Food Chem., 126(4):1821-35, 2011

Lillo, A.; Carvajal-Caiconte, F.; Nuñez, D.; Balboa, N. \& Alvear, M. Cuantificación espectrofotométrica de compuestos fenólicos y actividad antioxidante en distintos berries nativos del Cono Sur de América. RIA, 42(2):168-174, 2016.

Mihai, C.; Marghitas, L.; Dezmirean, D.; Chirila, F.; Moritz, R. \& Schlüns, $\mathrm{H}$. Interactions among flavonoids of propolis affect antibacterial activity against the honeybee pathogen Paenibacillus larvae. J. Invertebr. Pathol., 110(1):68-72, 2012.

Moreno, M.; Isla, M.; Sampietro, A. \& Vattuone, M. Comparison of the free radical-scavenging activity of propolis from several regions of Argentina. J. Ethnopharmacol., 71:109-14, 2000.

Naik, D.; Mujumdar, A. \& Vaidya, H. Anti-inflammatory activity of propolis from Maharashtra, India. J. Apicultural Res., 52(2):35-43, 2013.

Ngatu, N.; Saruta, T.; Hirota, R.; Eitoku, M.; Muzembo, B.; Matsui, T. \& Nangana, L. Antifungal efficacy of Brazilian green propolis extracts and honey on Tinea capitis and Tinea versicolor. European J. Integrative Medicine, 3(4):281-7, 2011.

Payá, M.; Halliwell, B. \& Hoult, J. Interactions of a series of coumarins with reactive oxygen species: scavenging of superoxide, hypochlorous acid and hydroxyl radicals. Biochem.Pharmacol., 44(2):205-14, 1992.

Premoli, G.; Laguado, P.; Díaz, N.; Romero, C.; Villareal, J. \& Gonzales, A. Uso del propóleo en odontología. Acta Odontológica Venezolana, 35(1), 2010.

Takos, A.; Jaffé, F.; Jacob, S.; Bogs, J.; Robinson, S. \& Walker, A. Lightinduced expression of a MYB gene regulates anthocyanin biosynthesis in red apples. Plant Physiology, 142(3):12-32, 2006.

Valenzuela-Barra, G.; Castro, C.; Figueroa, C.; Barriga, A.; Silva, X.; De las Heras, B. \& Delporte, C. Anti-inflammatory activity and phenolic profile of propolis from two locations in Región Metropolitana de Santiago, Chile. J. Ethnopharmacol., 168:37-44, 2015.

Zhanga, H.; Shan, Y.; Wu, Y.; Xu, C.; Yu, X; Zhao, J.; Yan, J. \& Shang, W. Berberine suppresses LPS-induced inflammation through modulating Sirt1/NF-kB signaling pathway in RAW264.7 cells. Int. Immunopharmacol., 52:93-100, 2017.

Dirección para correspondencia:

Adriana Vasconcellos

Departamento de Ciencias Básicas

Facultad de Medicina

Universidad de La Frontera

Av. Francisco Salazar 01145

Temuco

CHILE

Email: adriana.vasconcellos@ufrontera.cl

Recibido : 13-11-2017

Aceptado: 29-11-2017 